\title{
Effects of a series resistor on electron emission from a field emitter
}

\author{
J. W. Luginsland, A. Valfells, and Y. Y. Lau ${ }^{\text {a) }}$ \\ Department of Nuclear Engineering and Radiological Sciences, University of Michigan, \\ Ann Arbor, Michigan 48109-2104
}

(Received 1 July 1996; accepted for publication 19 August 1996)

\begin{abstract}
Universal curves are constructed that provide an immediate determination of the effect of a series resistor on the electron emission from a field emitter. These curves are applicable to both the low current and high current regime. The effects of space charge and of the series resistor are apparent from these curves, which are applicable to a large class of materials. An example is given to illustrate their use. (C) 1996 American Institute of Physics. [S0003-6951(96)02544-2]
\end{abstract}

Field emitters are an important, high brightness electron source for display and for generation of coherent radiation. ${ }^{1-3}$ Electron emission, from both metal and semiconductor field emitters, has been observed to follow the Fowler-Nordheim $(\mathrm{F}-\mathrm{N})$ relation, according to which the emission current density rises rapidly with voltage. This rapid rise in the emitter current with voltage leads to serious implication of the emitter stability (thermal, mechanical, and electrical), and an obvious way to improve the emitter stability is to add a series resistor to the emitter. ${ }^{4}$ However, the addition of a series resistor would result in a higher operating voltage, loss in efficiency, and much higher cost.

In this letter, we use a simple model to provide a quantitative analysis of the effects of a base resistor on the voltage-current $(V-I)$ characteristics of a single field emitter. Two features of the present work are noteworthy. First, we present a set of universal curves, from which the effects of a series resistor can immediately be determined once the F-N coefficients $A, B$, and the gap spacing $D$ are specified. Thus, these curves are applicable to a wide variety of field emitters. Second, our calculations take into account the effects of space charge that is present in the gap. The importance of the space charge and of the series resistor are apparent from the curves, as illustrated by the example given.

Before applying the theory to a specific device, let us first consider a planar model for the field emitter, driven by a dc voltage $V$ and connected to a series resistor $R$ at its base [Fig. 1(a)]. We assume that the field emitter emits according to the $\mathrm{F}-\mathrm{N}$ relation

$$
J=A E_{s}^{2} e^{-B / E_{s}},
$$

where $J$ is the emission current density, $E_{s}$ is the surface electric field, and the $\mathrm{F}-\mathrm{N}$ coefficients $A, B$ are taken to be known constants. Let $S$ be the area of the emitting surface, $D$ be the gap separation, and $V_{g}$ be the gap voltage. A lumped circuit element of impedance $Z$ is used to represent the field emitter [Fig. 1(b)]. Thus, we have

$$
V=V_{g}+I R=I(Z+R),
$$

where $I=J S$ is the emitter current. It is clear from the last expression that the base resistor $R$ will have a significant effect only when $R$ is of order $Z$ or larger.

\footnotetext{
a) Also with: Applied Physics Program, University of Michigan, Ann Arbor, Michigan 48109. Electronic mail: yylau@umich.edu
}

The field emitter impedance $Z$ depends nonlinearly on the current, which in turn depends on the electron dynamics within the gap. The determination of $Z$ requires a simultaneous solution to the equations of motion, the continuity equation, and the Poisson equation; with the $\mathrm{F}-\mathrm{N}$ relation being used as a boundary condition. The number of parameters in these equations may be substantially reduced if some scales are constructed in such a way that, in terms of these scales, all equations become dimensionless. Note that in so doing, the electron dynamics in the vacuum region may then be linked to the materials properties, which are represented by the $\mathrm{F}-\mathrm{N}$ coefficients $A$ and $B$ in the present formulation. It is obvious from Eq. (1) that the $\mathrm{F}-\mathrm{N}$ coefficient $B$ provides an electric field scale $E_{0}$, and the F-N coefficient $A$ provides a current density scale $J_{0}$. From these two scales, the time scale $\tau$, length scale $l$, and voltage scale $U$ follow. ${ }^{5}$ Specifically,

$$
\begin{aligned}
& E_{0}=B, \quad J_{0}=A B^{2}, \quad \tau=\epsilon_{0} E_{0} / J_{0}, \\
& l=e E_{0} \tau^{2} / m, \quad U=l E_{0},
\end{aligned}
$$

where $\epsilon_{0}$ is the free space permittivity, and $e$ and $m$ is, respectively, the electron charge and mass. In this letter all physical quantities, such as $e, J, I, V$, and $E_{s}$ are positive, by convention.

We shall use a bar to designate normalized quantities: $\bar{D}=D / l, \bar{J}=J / J_{0}, \bar{E}=E_{s} / E_{0}, \bar{V}=V / U, \bar{V}_{g}=V_{g} / U$. Then the $\mathrm{F}-\mathrm{N}$ relation (1) reads

$$
\bar{J}=\bar{E}^{2} e^{-1 / \bar{E}},
$$




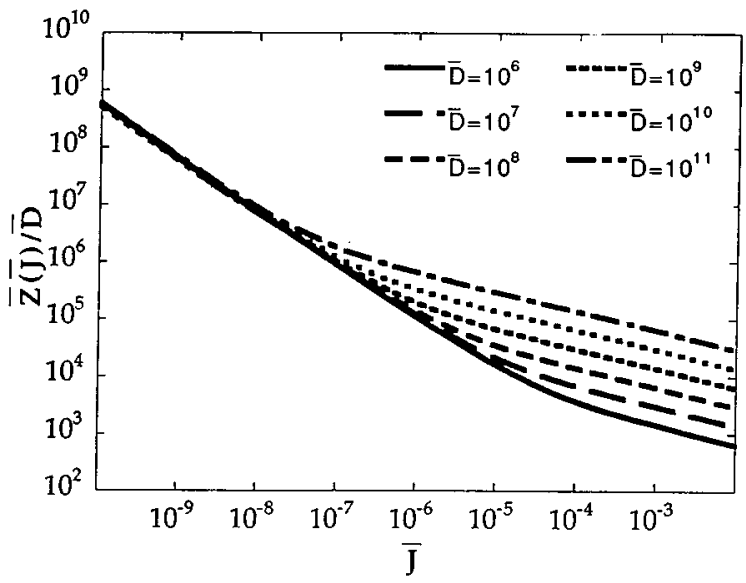

(a)

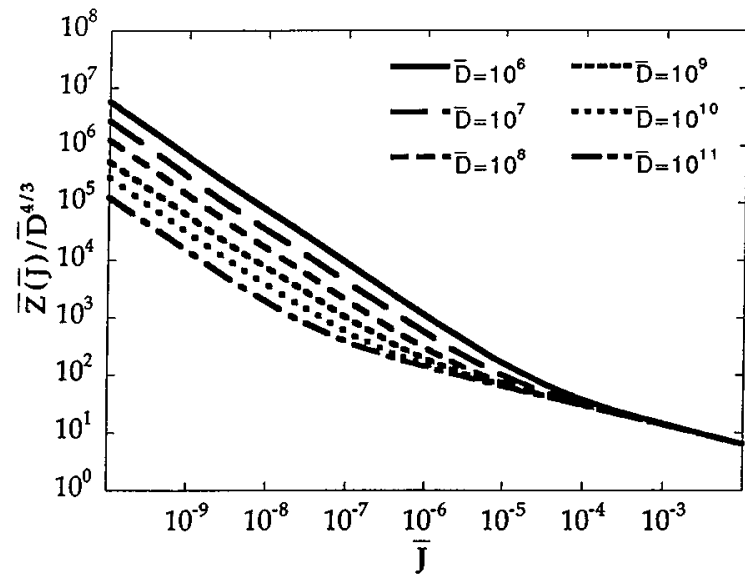

(b)

FIG. 2. (a) The plot of $\bar{Z} / \bar{D}$ vs $\bar{J}$ for various values of $\bar{D}$. The asymptote at low values of $\bar{J}$ is the Fowler-Nordheim relation in which the surface electric field is the vacuum field (i.e., space charge is unimportant). (b) The plot of $\bar{Z} / \bar{D}^{4 / 3}$ vs $\bar{J}$ for various values of $\bar{D}$. The asymptote at high values of $\bar{J}$ is the Child-Langmuir law (i.e., space charge effect is very important).

which becomes material independent. The circuit equation (2) may be written in either of the following forms (for reasons to be given shortly):

$$
\begin{aligned}
& \frac{\bar{V}}{\bar{J} \bar{D}}=\frac{\bar{Z}}{\bar{D}}+\frac{\bar{R}}{\overline{\bar{D}}}, \\
& \frac{\bar{V}}{\overline{\bar{J} \bar{D}^{4 / 3}}}=\frac{\bar{Z}}{\bar{D}^{4 / 3}}+\frac{\bar{R}}{\bar{D}^{4 / 3}},
\end{aligned}
$$

where the normalized impedances $\bar{Z}$ and $\bar{R}$ are defined by

$$
\bar{Z} \equiv \bar{V}_{g} / \bar{J}, \quad \bar{R} \equiv R \times\left(J_{0} S\right) / U
$$

The normalized impedance $\bar{Z}$ is a function of $\bar{J}$ only, once the normalized gap spacing $\bar{D}$ is specified. It may be obtained from the $V-I$ characteristics that was constructed from the simultaneous solution to the force law, continuity equation, Poisson equation, and the Fowler-Nordheim equation. ${ }^{5}$ Shown in Fig. 2(a) are the curves $\bar{Z} / \bar{D}$ as a function of $\bar{J}$ at several values of $\bar{D}$ [cf. Eq. (5a)]. From Fig. 2(a), we see that at low values of $\bar{J}$, the quantity $\bar{Z} / \bar{D}$ is independent of $\bar{D}$. The underlying reason is that at these low current densities, the space charge effects are unimportant, the nor- malized surface electric field $\bar{E}$ is approximately equal to the vacuum field $\bar{V}_{g} / \bar{D}$, in which case Eq. (4) may be inverted to yield $\bar{V}_{g} / \bar{D}$ as a function of $\bar{J}$. Thus, the function $\bar{Z} / \bar{D}$ $=\bar{V}_{g} / \overline{D J}$ is a function of $\bar{J}$ only, in this low current density limit. The divergence of the curves at higher values of $\bar{J}$ in Fig. 2(a) indicates that the space charge effects become important, i.e., when the surface electric field $\bar{E}$ may no longer be represented by the vacuum field $\bar{V}_{g} / \bar{D}$.

Another way to see the importance of the space charge effects at high current densities is to plot $\bar{Z} / \bar{D}^{4 / 3}$ as a function of $\bar{J}$ [cf. Eq. (5b)]. These curves are shown in Fig. 2(b) where we see that at high values of $\bar{J}$, all curves converge to the same asymptote, regardless of $\bar{D}$. Specifically, this high current asymptote reads $\bar{Z} / \bar{D}^{4 / 3}=(81 / 32 \bar{J})^{1 / 3}$, which is simply the Child-Langmuir law, $\bar{V}_{g}=\left(81 \bar{J}^{2} \bar{D}^{4} / 32\right)^{1 / 3}$, written in normalized form. ${ }^{6}$

Thus, Eq. (5a) and Fig. 2(a) [equivalently, Eq. (5b) or Fig. 2(b)] provide an immediate assessment of the importance of the series resistor $R$ at various levels of emitter current. In other words, the curves in Figs. 2(a) and 2(b) provide the value of the series resistor $R$ that would yield a matched load to $Z$ [Fig. 1(b)], at various operating current levels as measured by $\bar{J}$. Note also that these curves are "universal,"' in the sense that each one of them is fixed once $\bar{D}$ is specified.

To apply the above planar model to a realistic field emitter with a field enhancement factor $\beta$ ( $\beta=1$ for the planar model), we assume that we may simply replace the electric field scale $E_{0}=B / \beta$ whereas all other scale lengths defined in Eq. (3) remain unchanged. ${ }^{7}$

As an example, consider a field emitter with work function $\phi=4 \mathrm{eV}, D=0.5 \mu \mathrm{m}, S=3 \times 10^{-16} \mathrm{~m}^{2}$, and $\beta=20$ [See Ref. 7]. In the SI units, this work function gives Fowler-Nordheim coefficients $A=4.72 \times 10^{-5}$ and $B=5.2$ $\times 10^{10}$, upon using the frequently used expressions of $A, B$ in terms of $\phi$ [See, e.g., Ref. 5]. The electric field scale $E_{0}=B / \beta=2.6 \times 10^{9} \mathrm{~V} / \mathrm{m}$, the current density sale $J_{0}=A B^{2}$ $=1.27 \times 10^{17} \mathrm{~A} / \mathrm{m}^{2}$, and the other scales in Eq. (3) are $\tau=1.81 \times 10^{-19} \mathrm{~s}, l=1.49 \times 10^{-17} \mathrm{~m}$, and $U=3.87 \times 10^{-8}$ $\mathrm{V}$. As these scales are obtained from a mathematical construction, they do not have a ready physical interpretation; but their use enables us to reduce the numerous independent parameters into a single one, $\bar{D}$, as shown in Fig. 2 . In the present example, $\bar{D}=D / l=3.36 \times 10^{10}$. If we are interested in a current of $I=0.5 \mu \mathrm{A}$ being drawn from such a field emitter, the value of $J=I / S=1.67 \times 10^{9} \mathrm{~A} / \mathrm{m}^{2}$, and the normalized current density becomes $\bar{J}=J / J_{0}=1.31 \times 10^{-8}$. For these values of $\bar{J}$ and $\bar{D}$, Fig. 2(a) shows that the space charge effects are unimportant and that $\bar{Z} / \bar{D} \approx 10^{7}$. This gives $\bar{Z}$ $=3.36 \times 10^{17}$, and therefore $\bar{V}_{g}=\overline{Z J}=4.4 \times 10^{9}$. This implies a gap voltage $V_{g}=\bar{V}_{g} U=170 \mathrm{~V}$ and the emitter impedance $Z=V_{g} / I=3.4 \times 10^{8} \Omega$. In the presence of a series resistor $R$, the external voltage required is $V=170 \mathrm{~V}+R \times 0.5 \mu \mathrm{A}$. The base resistor will exercise a negligible effect (at $0.5 \mu \mathrm{A}$ ) only if $R \ll 340 \mathrm{M} \Omega$. The numerical values of these impedances may seem very large. Recall, however, that these values are for a single tip, while the number of tips, which are in parallel, is very large in practice.

For other values of emitter current $I$, we can use a simi- 


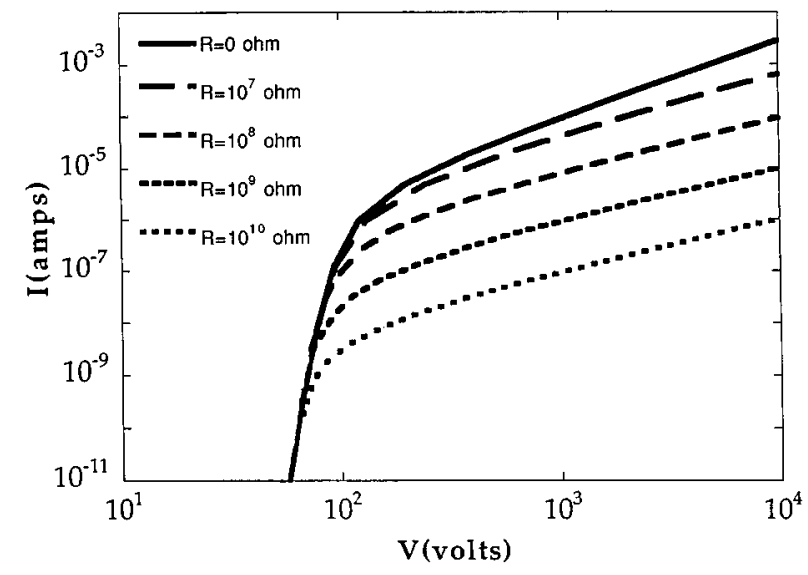

FIG. 3. The $I-V$ characteristic at several values of the series resistor $R$. Field emitter parameters: $\phi=4 \mathrm{eV}, D=0.5 \mu \mathrm{m}, S=3 \times 10^{-16} \mathrm{~m}^{2}$, and $\beta=20$.

lar procedure to deduce the external voltage $V$ required. $V-I$ characteristics constructed this way are shown in Fig. 3 for the above example, for various series resistors. From Fig. 3, we see the obvious fact that for the emitter to emit at a certain level of current, the required dc voltage $V$ increases with the resistor $R$. Note also that, at low voltages, the current is exponentially small and, as a result, the voltage across the resistor is also exponentially small and the resistor $R$ then has a negligible effect on the $I-V$ characteristics, as shown in Fig. 3 when $V \ll 100 \mathrm{~V}$. The $I-V$ characteristics in this low voltage regime is simply the $\mathrm{F}-\mathrm{N}$ relation in which the surface electric field is $V / D$, independent of resistor. In the limit of high voltages, the situation changes dramatically, depending on the value of the resistance. When $R$ is low, (the limiting case being $R \rightarrow 0$ ), the gap voltage $V_{g}$ becomes the dc supply voltage $V$ which, at high values, yields the ChildLangmuir limiting current. The $R=0$ curve in Fig. 3 then shows the transition from the $\mathrm{F}-\mathrm{N}$ relation to the ChildLangmuir law as the voltage increases. At a high value of resistor, the current $I$ approaches $V / R$ at high voltages, since $Z$ would be small compared with $R$ [cf. Eq. (2)]. Once more, the relative importance of the resistor is measured by $R / Z$, and the latter quantity is current dependent [cf. Fig. 2].

The general treatment given in this letter is applicable to all emitters as long as the emission law is governed by the $\mathrm{F}-\mathrm{N}$ relation. The adoption of the planar model is expected to be reliable when the effect of space charge is small. Less certain is when the space charge effect becomes substantial, in which case the use of the planar model and of a single local field enhancement factor $(\beta)$ adopted here may be questionable. What is given here is then an identification of the regimes where the series resistor and the space charge effects are significant, as well as a quantitative assessment of their relative importance.

This work was motivated by several discussions with Bruce Gnade, George Haddad, Johnson Lin, Stella Pang, and J. P. Sun. It was supported by DoD/AASERT and by NRL/ ONR.

${ }^{1}$ I. Brodie and P. R. Schwoebel, Proc. IEEE 82, 1006 (1994).

${ }^{2}$ J. P. Calame, H. F. Gray, and J. L. Shaw, J. Appl. Phys. 73, 1485 (1993).

${ }^{3}$ R. K. Parker and R. H. Abrams, International Electron Devices Meeting, Technical Digest (Institute of Electrical and Electronic Engineers, New York, 1990), p. 7.1.1 [IEEE Catalog No. 90CH2865-4].

${ }^{4}$ J. D. Levine, J. Vac. Sci. Technol. B 13, 474 (1995); 14, 2008 (1996); J. D. Levine et al., ibid. 13, 553 (1995).

${ }^{5}$ Y. Y. Lau, Y. Liu, and R. K. Parker, Phys. Plasmas 1, 2082 (1994).

${ }^{6}$ The Child-Langmuir Law is also obtained at very high current density for the case of nonzero surface electric field that is required for field emission. This observation is implicit in Ref. 5. For details, see Youfan Liu, Ph.D. thesis, University of Michigan, Ann Arbor, Michigan, 1996.

${ }^{7}$ One way to apply the planar model to a realistic emitter is to determine the unknown quantities $\beta$ and $S$ semiemperically (assuming that $A, B$, and the tip anode spacing $D$ are given) from the $F-N$ plot, which is a plot of $\log$ $\left(I / V_{g}^{2}\right)$ versus $\left(1 / V_{g}\right)$. This plot is a straight line with a negative slope in the low voltage regime [cf. Eq. (4)]. The measured slope of this straight line gives $(-B D / \beta)$, which yields $\beta$ as $B$ and $D$ are given, while the measured intercept of this straight line with the ordinate gives $\log$ $\left(S A \beta^{2} / D^{2}\right)$, which yields $S$. 